\title{
The Fairest Core in Cooperative Games with Transferable Utilities
}

\author{
Tri-Dung Nguyen \\ School of Mathematics, Building 54, Room 10005 \\ University of Southampton, Southampton, UK, SO17 $37 J$
}

\begin{abstract}
The core and the Shapley value are important solution concepts in cooperative game theory. While the core is designed for the stability of the game, the Shapley value aims for fairness among the players. However, the Shapley value might not lie within the core and a core solution might not be 'fair'. We introduce a new solution concept called the 'fairest core', one that aims for both stability and fairness. We show attractive properties of the fairest core.
\end{abstract}

Keywords: Cooperative games, the core, the Shapley value, stability, fairness.

\section{Introduction and Literature Review}

\subsection{Cooperative Games and Solution Concepts}

Let $n$ be the number of players and let $\mathcal{N}=\{1,2, \ldots, n\}$ be the set of all the players. A coalition $\mathcal{S}$ is a subset of the players, i.e. $\mathcal{S} \subseteq \mathcal{N}$. The characteristic function $v: 2^{\mathcal{N}} \mapsto \mathbb{R}$ maps each coalition $\mathcal{S}$ to a real number with $v(\mathcal{S})$ representing the payoff that coalition $\mathcal{S}$ is guaranteed to obtain if all players in $\mathcal{S}$ collaborate, no matter what the remaining players do. A solution of the game $\boldsymbol{x}=\left(x_{1}, x_{2}, \ldots, x_{n}\right)$ is a way to distribute the reward among the players, with $x_{i}$ being the share for player $i$. Let us denote $\boldsymbol{x}(\mathcal{S})=\sum_{i \in \mathcal{S}} x_{i}$. For each solution $\boldsymbol{x}$, the excess value of a coalition $\mathcal{S}$ is defined as $e(\mathcal{S}, \boldsymbol{x})=v(\mathcal{S})-\boldsymbol{x}(\mathcal{S})$ which can be viewed as the level of dissatisfaction the players in coalition $\mathcal{S}$ feel over the proposed solution $\boldsymbol{x}$. Solution concepts for cooperative games include:

- An imputation is a solution $\boldsymbol{x}$ that satisfies $\boldsymbol{x}(\mathcal{N})=v(\mathcal{N})$ and $x_{i} \geq v(\{i\}), \forall i \in \mathcal{N}$.

- The core of the game is the set of all imputations $\boldsymbol{x}$ such that $e(\mathcal{S}, \boldsymbol{x}) \leq 0, \forall \mathcal{S} \subset \mathcal{N}$. The $\epsilon$-core is defined as the set of all imputations $\boldsymbol{x}$ such that $e(\mathcal{S}, \boldsymbol{x}) \leq \epsilon, \forall \mathcal{S} \subset \mathcal{N}$.

- The least core is the non-empty $\epsilon$-core with $\epsilon$ being the smallest.

- The Shapley value is defined as: $\phi=\left\{\phi_{1}, \ldots, \phi_{n}\right\}$ where

$$
\phi_{i}=\sum_{\mathcal{S} \subset \mathcal{N}} \frac{|\mathcal{S}| !(n-|\mathcal{S}|-1) !}{n !}(v(\mathcal{S} \cup i)-v(\mathcal{S})),
$$

i.e. the Shapley value of player $i$ is the weighted average of the marginal contributions that player $i$ has on all possible coalitions.

\subsection{Properties and Issues with the Core and the Shapley Value}

Table 1 shows a summary of the properties of three important solution concepts in cooperative games, namely the core, the least core and the Shapley value. The first column includes desirable properties of the payoff distributions.

- Efficiency: All three solution concepts are efficient as the entire payoff of the grand coalition is distributed to all the players, i.e. $\boldsymbol{x}(\mathcal{N})=v(\mathcal{N})$.

Email address: T.D.Nguyenesoton.ac.uk. Telephone: +0044 02380597759 (Tri-Dung Nguyen) 


\begin{tabular}{|c|c|c|c|}
\hline & Core & Least core & Shapley value \\
\hline Efficiency & Yes & Yes & Yes \\
\hline Existence & Not guaranteed & Yes & Yes \\
\hline Uniqueness & Not likely & Not likely & Yes \\
\hline Stability & Yes & Yes & Might not \\
\hline 'Fairness' & Not likely & Not likely & Yes \\
\hline
\end{tabular}

Table 1: Properties of the core, the least core and the Shapley value.

- Existence and uniqueness: While the Shapley value exists and is unique, the core might not exist in some games. In that case, the least core is introduced as the set of solutions with the least dissatisfaction, i.e. those with the worst excess value being minimized. However, the core, if it exists, and the least core are often non-unique. This might lead to ambiguity, and it is not desirable in real applications because different stakeholders might wish to use different solutions.

- Stability: The core, if it exists, is defined to ensure the stability of the game, in the sense that no group of players has the incentive to break out of the grand coalition, because the total share allocated to them is at least the total payoff that they can obtain by forming a coalition themselves. For games with an empty core, the least core is defined in a similar way to minimise the worst dissatisfaction. As the core and the least core are defined to avoid the possibility of groups of players breaking out of the grand coalition, they are referred to as solution concepts with the stability property. The Shapley value, on the other hand, does not aim for this, and hence it is possible to have unstable Shapley values. We can find many real examples (e.g. in setting ATM interchange fee [2]) and simulated examples (see Section 4) in which the Shapley values are unstable.

- Fairness w.r.t. dummy players: The core, the least core, and the Shapley value all suggest that a dummy player who contributes nothing (or a constant value) to all the coalitions receives a share of zero (or the same constant value).

- Fairness w.r.t. symmetry: The Shapley value ensures symmetry among the players in the sense that players with the same vectors of marginal contributions should receive the same shares. However, most of the solutions in the core and the least core do not have this property.

- Fairness w.r.t. monotonicity: The Shapley value ensures monotonicity among the players, in the sense that a player whose marginal contributions are consistently greater than those of another with respect to any coalition should receive a higher share.

- Fairness w.r.t. sparsity: The Shapley value ensures that a player who has any positive contribution to any coalition should receive a positive share, as long as the player does not make any negative contribution to others. Nevertheless, not all solutions in the core and the least core have this property.

- Additivity: A nice property of the Shapley value is the additivity property. Given two cooperative games $G_{1}\left(\mathcal{N}, v_{1}\right)$ and $G_{2}\left(\mathcal{N}, v_{2}\right)$ that are defined on the same set of players, the Shapley value of the combined game $G_{3}\left(\mathcal{N}, v_{3}=\right.$ $\left.v_{1}+v_{2}\right)$ is equal to the sum of the Shapley values of the two games $G_{1}$ and $G_{2}$. Shapley [9] proves that three axioms on symmetry, additivity and dummy uniquely define the Shapley value. The additivity property is desirable in situations where the characteristic function of the game of interest is a weighted average of several characteristic functions. One such example is the case of a characteristic function that is defined as the expectation over a number of scenarios, each corresponding to a particular value function. In this case, the Shapley value of the stochastic game is simply the average of the Shapley value of the individual scenarios, i.e. $E[\phi[G(\mathcal{N}, \tilde{v})]=\phi[G(\mathcal{N}, E[\tilde{v}])]$.

\section{The Fairest Core and the Fairest Least Core}

We have seen in subsection 1.2 that the core, the least core and the Shapley can be grouped into two categories, with the first two solution concepts aiming for stability, while the last one ensures fairness among the players. To apply cooperative game theory successfully to a real application, it would be ideal if the core exists and the Shapley value lies within the 
core. In this case, choosing the Shapley value would be ideal, since the payoff distribution is unique, fair and stable. It has been shown in [10] that every convex game has a non-empty core and the Shapley value lies within the core. However, it is unfortunate that this is not true for all games. In fact, we will show in an experiment in Section 4 that the Shapley value does not lie within the core in $77 \%$ of the random minimum spanning tree games generated. In this case, the question of choosing which solution to balance between fairness and stability is a tricky one. The goal of this manuscript is to find a solution concept that has desirable properties and at the same time is not too difficult to compute. We introduce the concept of the fairest core, a unique and stable solution that also has some fairness properties. This solution is the one that is closest to the Shapley value (to be fair), belongs to the core (to be stable), and is formally defined as follows:

Definition 2.1. Given a game $G(\mathcal{N}, v)$ with non-empty core, the fairest core is a solution in the core that has the closest Euclidean distance to the Shapley value, i.e. the fairest core is an optimal solution of the following optimization problem:

$$
\begin{array}{ll}
\min _{\boldsymbol{x}} & \|\boldsymbol{x}-\boldsymbol{\phi}\| \\
\text { s.t. } & \boldsymbol{x}(\mathcal{N})=v(\mathcal{N}), \\
& \boldsymbol{x}(\mathcal{S}) \geq v(\mathcal{S}), \forall \mathcal{S} \subset \mathcal{N} .
\end{array}
$$

In Definition 2.1, the fairest core is defined on a game with non-empty core. For general cases, we extend this to the concept of the fairest least core, which is defined as follows:

Definition 2.2. Given a game $G(\mathcal{N}, v)$, the fairest least core is a solution in the least core that has the closest Euclidean distance to the Shapley value, i.e. the fairest least core is an optimal solution of the following optimization problem:

$$
\begin{array}{ll}
\min _{\boldsymbol{x}} & \|\boldsymbol{x}-\boldsymbol{\phi}\| \\
\text { s.t. } & \boldsymbol{x}(\mathcal{N})=v(\mathcal{N}), \\
& \boldsymbol{x}(\mathcal{S}) \geq v(\mathcal{S})-\epsilon^{*}, \forall \mathcal{S} \subset \mathcal{N},
\end{array}
$$

where $\epsilon^{*} \geq 0$ is the worst excess level for solutions in the least core.

Notice that if the core is non-empty, the two concepts of the fairest core and the fairest least core coincide. In order to compute the fairest least core, we need to compute the least core first. Then we solve the fairest core of a new game whose characteristic function is offset by $\epsilon^{*}$ for all but the grand coalition.

\section{Properties of the Fairest Core and the Fairest Least Core}

The first two main results of this research concern the properties of the fairest core and the fairest least core as stated in the following theorems:

Theorem 1. For games with non-empty core, the fairest core exists and has the following properties: (a) It is unique, (b) Dummy players receive zero shares, (c) It is symmetric in the sense that two players with the same vectors of marginal contributions should receive the same shares, and (d) It is monotone in the sense that if the marginal contribution of player $i$ is consistently larger than that of player $j$, i.e. $v(\mathcal{S} \cup\{i\}) \geq v(\mathcal{S} \cup\{j\}), \forall \mathcal{S} \subset \mathcal{N}$, then the share of player $i$ should be larger than that of player $j$.

Before presenting the proof of Theorem 1, let us explore its implications. First, since the fairest core belongs to the core, it has all the properties of the core, including stability. In addition, it is unique, which means there is no ambiguity when we refer to this solution concept. It also shares some fairness properties with the Shapley value, namely dummy, symmetry and monotonicity. The monotonicity property implies that the fairest core (and the fairest least core if the core is empty) preserves the ranking between the players if their contributions can be clearly distinguishable. This property is desirable in situations where we want to apply cooperative game theory to find the importance or the ranking among the players, such as in terrorist detection (see [7] for details about the application.)

Proof of Theorem 1:

a. Uniqueness: Since the objective function is strictly convex, the optimal solution, if it exists, is unique.

b. Dummy player gets zero: Since the fairest core belongs to the core, we have $0=v(\{i\}) \leq x_{i}=\boldsymbol{x}(\mathcal{N})-\boldsymbol{x}(\mathcal{N} \backslash i) \leq$ $v(\mathcal{N})-v(\mathcal{N} \backslash i)=0$ and hence $x_{i}=0$. 
c. Symmetry: Let $\boldsymbol{x}$ be the fairest core. Consider two players $i, j$ and suppose that the vector of marginal contributions of player $i$ is equal to that of player $j$, i.e. $v(\mathcal{S} \cup\{i\})=v(\mathcal{S} \cup\{j\}), \forall \mathcal{S} \subset \mathcal{N}$. We will prove that $\boldsymbol{x}_{i}=\boldsymbol{x}_{j}$.

Due to the symmetry of the characteristic function on players $(i, j)$, the constraint set should be symmetric between the two players. In addition, $\phi_{i}=\phi_{j}$ since the two players have symmetric marginal contributions. Therefore the optimisation problem shown in model (1) is symmetric over the two players. This means a new solution $\boldsymbol{y}$ that is constructed by swapping the values of $y_{i}=x_{j}$ and $y_{j}=x_{i}$ while fixing $y_{k}=x_{k}, \forall k \notin\{i, j\}$ will also be an optimal solution. Since the problem has a unique solution, we must have $\boldsymbol{y}=\boldsymbol{x}$. Thus, $x_{i}=x_{j}$.

d. Monotonicity: Consider two players $i, j$ and suppose that the marginal contribution of player $i$ is consistently larger than that of player $j$, i.e. $v(\mathcal{S} \cup\{i\}) \geq v(\mathcal{S} \cup\{j\}), \forall \mathcal{S} \subset \mathcal{N}$. We will show that $\boldsymbol{x}_{i} \geq \boldsymbol{x}_{j}$.

Suppose on contradiction that $x_{j}-x_{i}=\delta>0$. For any $\epsilon$ such that $0<\epsilon<\delta$, consider another solution $\boldsymbol{y}$ that is constructed by setting $y_{i}=x_{i}+\epsilon, y_{j}=x_{j}-\epsilon$, while fixing $y_{k}=x_{k}, \forall k \notin\{i, j\}$. We will show that $\boldsymbol{y}$ belongs to the core and that $\|\boldsymbol{y}-\boldsymbol{\phi}\|<\|\boldsymbol{x}-\phi\|$ which means $\boldsymbol{x}$ is not the fairest core, and hence a contradiction.

We have $\boldsymbol{y}(\mathcal{N})=\boldsymbol{x}(\mathcal{N})=v(\mathcal{N})$. We need to show $\boldsymbol{y}(\mathcal{S}) \geq v(\mathcal{S}), \forall \mathcal{S} \subset \mathcal{N}$. There are four cases that a coalition $\mathcal{S}$ can fall in:

- Case 1: $\mathcal{S} \ni\{i, j\}$, i.e. coalition $\mathcal{S}$ contains both players, we have $\boldsymbol{y}(\mathcal{S})=\boldsymbol{x}(\mathcal{S}) \geq v(\mathcal{S})$.

- Case 2: $\mathcal{S} \not \supset\{i, j\}$, i.e. coalition $\mathcal{S}$ contains none of the two players, we also have $\boldsymbol{y}(\mathcal{S})=\boldsymbol{x}(\mathcal{S}) \geq v(\mathcal{S})$.

- Case 3: $\mathcal{S} \ni i$ and $\mathcal{S} \not \ngtr j$, we have $\boldsymbol{y}(\mathcal{S})=\boldsymbol{x}(\mathcal{S})+\epsilon \geq v(\mathcal{S})+\epsilon>v(\mathcal{S})$.

- Case 4: $\mathcal{S} \ni j$ and $\mathcal{S} \not \supset i$, we have,

$$
\begin{aligned}
\boldsymbol{y}(\mathcal{S}) & =\boldsymbol{x}(\mathcal{S})-\epsilon \\
& =\boldsymbol{x}(\mathcal{S} \backslash j)+x_{j}-\epsilon \\
& =\boldsymbol{x}(\mathcal{S} \backslash j \cup\{i\})+x_{j}-x_{i}-\epsilon \\
\geq & v(\mathcal{S} \backslash j \cup\{i\})+x_{j}-x_{i}-\epsilon \\
\geq & v(\mathcal{S})+x_{j}-x_{i}-\epsilon>v(\mathcal{S}) .
\end{aligned}
$$

Here inequality (3) is due to $\boldsymbol{x}$ belonging to the core, inequality (4) comes from the assumption that the marginal contributions of player $i$ are greater than those of player $j$, and from the choice of $\epsilon<\delta=x_{j}-x_{i}$.

To complete the proof, we need to show that $\|\boldsymbol{y}-\boldsymbol{\phi}\|<\|\boldsymbol{x}-\boldsymbol{\phi}\|$. We have:

$$
\begin{aligned}
(\boldsymbol{y}-\boldsymbol{\phi})^{t}(\boldsymbol{y}-\boldsymbol{\phi}) & =\left(x_{i}+\epsilon-\phi_{i}\right)^{2}+\left(x_{j}-\epsilon-\phi_{j}\right)^{2}+\sum_{k \notin\{i, j\}}\left(x_{k}-\phi_{k}\right)^{2} \\
& =\sum_{k}\left(x_{k}-\phi_{k}\right)^{2}+2 \epsilon\left(\epsilon+\left(x_{i}-x_{j}\right)-\left(\phi_{i}-\phi_{j}\right)\right) \\
& \left.=(\boldsymbol{x}-\phi)^{t}(\boldsymbol{x}-\phi)-2 \epsilon(\delta-\epsilon)-2 \epsilon\left(\phi_{i}-\phi_{j}\right)\right) \\
& <(\boldsymbol{x}-\boldsymbol{\phi})^{t}(\boldsymbol{x}-\boldsymbol{\phi}) .
\end{aligned}
$$

Here inequality (5) comes from the fact that $\delta>\epsilon>0$ and that $\phi_{i} \geq \phi_{j}$.

We can prove the same result for the fairest least core as formally stated in the following theorem:

Theorem 2. For general games, the fairest least core exists and has all the four properties listed in Theorem 1, namely Uniqueness, Symmetry, Dummy, and Monotonicity.

Proof: Consider a game $G_{1}\left(\mathcal{N}, v_{1}\right)$ with $\epsilon^{*}$ being the worst deficit of the solutions in the least core. We define a new game $G_{2}\left(\mathcal{N}, v_{2}\right)$ with $v_{2}(\mathcal{S})=v_{1}(\mathcal{S})-\epsilon^{*}, \forall \mathcal{S} \neq \mathcal{N}$ and $v_{2}(\mathcal{N})=v_{1}(\mathcal{N})$. Since the change in the characteristic function is taken in the same manner for all the players, and since the payoff of the grand coalition is still the same, the Shapley value of $G_{2}$ is the same as that of $G_{1}$. The core of $G_{2}\left(\mathcal{N}, v_{2}\right)$ is exactly the same as the least core of $G_{1}\left(\mathcal{N}, v_{1}\right)$ due to its construction and hence is non-empty. In addition, the fairest core of $G_{2}\left(\mathcal{N}, v_{2}\right)$ is the same as the fairest least core of $G_{1}\left(\mathcal{N}, v_{1}\right)$ as they are unique solutions of the same optimization model (i.e. they are the projections of the same Shapley value on the same convex set). Thus, the fairest least core of $G_{1}\left(\mathcal{N}, v_{1}\right)$ has many properties of the fairest core of $G_{2}\left(\mathcal{N}, v_{2}\right)$ which include: Existence, Uniqueness, Dummy, Symmetry and Monotonicity. 


\subsection{Computational Complexity}

The next result concerns the computational complexity of the fairest core and the fairest least core. For games with a number of players less than 20, we can find the core (or the least core), the Shapley value and the fairest core (or the fairest least core) very easily. For larger games, finding the fairest core involves first finding the Shapley value as an input and then solving a strictly convex quadratic optimization problem over a polyhedron that has the same structure as the core, i.e. having an exponentially large number of constraints. If the Shapley value is given, e.g. in analytical forms based on the special structure of the game, we will show in Theorem 3 that computing the fairest core is no harder than finding the core. In fact, in our numerical result in Section 4, we show that solving the quadratic program in model (1) requires less time than that for finding the core.

Theorem 3. If the Shapley value $\phi$ is given and if the separation problem $\max _{\mathcal{S} \subset \mathcal{N}}\{v(\mathcal{S})-\boldsymbol{x}(\mathcal{S})\}$ can be solved in polynomial time for any given $\boldsymbol{x}$, then the fairest core can be computed in polynomial time.

We notice two existing results. First of all, a convex quadratic problem can be solved in polynomial time on the problem size by using various methods such as an ellipsoid method [6] and interior point methods [8]. However, in our case, the number of constraints is exponentially large and hence we cannot apply these results. Secondly, if the objective function in the formulation of the fairest core shown in model (1) was linear, then this theorem is a result of the ellipsoid method applying to linear programming [4]. As the ellipsoid method was originally developed by Naum Shor, Arkadi Nemirovski and David B. Yudin for general convex optimisation problems, Theorem 3 states a standard result. Notice also that finding the core is polynomial time solvable if, and only if, finding a separating hyperplane can be done in polynomial time (see Theorem 6.4.9 in [4]). Thus, the fairest core can be computed in polynomial time if the core can be computed in polynomial time.

Computing the fairest core requires the input Shapley value which might be difficult to compute for large games ( $n \geq$ 25). In this case, sampling methods are often used to approximate the Shapley value. With the approximation error from the input Shapley value, we would also expect an approximation error on the output fairest core. It is interesting, however, to note that the approximation error in the output is shrunk compared to that from the input due to the projection, as is formally stated in Theorem 4 . In addition, under some assumptions on the approximated Shapley value, the approximated fairest core preserves all the properties listed in Theorem 1.

Theorem 4. Let $\phi$ be the Shapley value and $\tilde{\boldsymbol{\phi}}$ be its approximation. Let $\boldsymbol{x}$ and $\tilde{\boldsymbol{x}}$ be the corresponding 'truth' and approximated fairest cores. Then we have:

a. $\|\boldsymbol{x}-\tilde{\boldsymbol{x}}\| \leq\|\tilde{\phi}-\phi\|$.

b. If $\tilde{\phi}$ is efficient, i.e. $\tilde{\phi}(\mathcal{N})=v(\mathcal{N})$, and preserves the ranking of $\phi$, i.e. for any pair of player $i, j$ we have $\tilde{\phi}_{i} \geq \tilde{\phi}_{j}$ iff $\phi_{i} \geq \phi_{j}$, then the approximated fairest core $\tilde{\boldsymbol{x}}$ has all the attractive properties listed in Theorem 1.

Proof: Part (a) is a standard result in projection theory, where the projection on a convex body always shrinks the distance. For part (b), since the only place in the proof of Theorem 1 that involves $\phi$ is in inequality (5) and since that derivation only requires the efficiency and the ranking of $\phi$ to be preserved, part (b) of Theorem 4 is proved.

Notice that if we replace $\phi$ with $(v(\mathcal{N}) / n) \boldsymbol{e}$, which is a vector of all one scaled up by $(v(\mathcal{N}) / n)$, i.e. for situations when we have no idea about the Shapley value and hence take the equal share vector as the best guess, we can still show that all the properties in Theorem 1 hold.

\subsection{Bounds on the Fairest Core}

The next result concerns the bounds on the fairest core. Given any solution $\boldsymbol{x}$ within the core, we have $x_{i} \geq v(\{i\})$ and hence $v(\{i\})$ is a lower bound of $x_{i}$. Consider a simple example with two players and with the characteristic function $v(\{1\})=v(\{2\})=0, v(\{1,2\})=10$. In this case, we have $x_{1} \geq v(\{1\})=0$. Although this lower bound is tight for the core, it is not very informative for the fairest core and should be strengthened. Similarly, given that $\boldsymbol{x}$ belongs to the core, we can also derive an upper bound of $x_{i} \leq v(\mathcal{N})-v(\mathcal{N} \backslash i)$. In this same simple example, we have $x_{1} \leq$ $v(\{1,2\})-v(\{2\})=10$. This upper bound is again tight for the core, but is not very informative for the fairest core and should also be strengthened.

Let us define $\bar{\delta}_{i}:=\max _{\mathcal{S} \neq \mathcal{N}}\{v(\mathcal{S})-v(\mathcal{S} \backslash i)\}$, i.e. $\bar{\delta}_{i}$ is the maximum marginal contribution of player $i$ to all coalitions except for the grand coalition (the exception of the grand coalition is to avoid the obvious upper bound that we have already 
seen in the previous paragraph). Let $\underline{\delta}_{i}:=\min _{\mathcal{S} \neq \emptyset}\{v(\mathcal{S} \cup\{i\})-v(\mathcal{S})\}$ be the minimum contribution of player $i$ to any coalition other than the empty set (the exception of the empty set is also to strengthen the lower bound). Then we have the following theorem:

Theorem 5. The share of player $i$ in the fairest core is at most $\max \left(\bar{\delta}_{i}, \phi_{i}\right)$ and at least $\min \left(\underline{\delta}_{i}, \phi_{i}\right)$.

Before presenting the proof, let us explore the implication of this result through the aforementioned simple example. In this case, the Shapley value of the game is $\phi=(5,5)$. For the first player, we have $\bar{\delta}_{1}:=\max _{\mathcal{S} \neq \mathcal{N}}\{v(\mathcal{S})-v(\mathcal{S} \backslash 1)\}=$ $v(\{1\})-v(\emptyset)=0$. Thus, the new upper bound on the share of player 1 is $\max \left(\bar{\delta}_{1}, \phi_{1}\right)=5$. We notice that this upper bound is smaller than the upper bound $v(\{1,2\})-v(\{2\})=10$ that we obtained earlier using the fact that $\boldsymbol{x}$ belongs to the core. We can also calculate $\underline{\delta}_{1}:=\min _{\mathcal{S} \neq \emptyset}\{v(\mathcal{S} \cup\{1\})-v(\mathcal{S})\}=v(\{1,2\})-v(\{2\})=10$ and obtain $\min \left(\underline{\delta}_{1}, \phi_{1}\right)=5$. This lower bound is also larger than the lower bound $v(\{1\})=0$ that we derived earlier. Putting all together, we obtain $5 \leq x_{1} \leq 5$ which results in $x_{1}=5$. Similarly, we can also work out $x_{2}=5$. The fairest core is therefore $\boldsymbol{x}=(5,5)$.

To understand the effectiveness of these bounds for general games, let us first discuss about the sensitivity analysis of the core. Given any core solution $\boldsymbol{x}$ of an original game $G(\mathcal{N}, v)$, if we increase $v(\mathcal{N})$ by a positive amount $\Delta$, then we can construct core members of the new game by adding $\boldsymbol{x}$ with any division of the extra payoff $\Delta$ among $n$ players. This means the core of the new game will contain solutions where a player might receive anything between zero and the entire extra payoff $\Delta$, and that is unfair. Let us see how the upper bound $\max \left(\bar{\delta}_{i}, \phi_{i}\right)$ can reduce the 'unfairness' in the fairest core. We can see that $\bar{\delta}_{i}$ is independent of $\Delta$ while the Shapley value $\phi_{i}$ is increased by $\Delta / n$ which is the even distribution of $\Delta$ among all the players. Thus the upper bound, if increased, is only changed by the same amount. This partially explains why the fairest core has the properties listed in Theorem 1. Notice that if the marginal contributions of player $i$ are constant $\delta_{i}$, then $\phi_{i}=\bar{\delta}_{i}=\underline{\delta}_{i}=\delta_{i}$. In this case, the upper bound and the lower bounds are both equal to $\delta_{i}$ and hence $x_{i}=\delta_{i}$. This same result can also be derived by using the fact that $\boldsymbol{x}$ lies within the core.

Proof of Theorem 5:

Upper bound: Let $\boldsymbol{x}$ be the fairest core. Suppose on contradiction that $\epsilon=\min \left(x_{i}-\bar{\delta}_{i}, x_{i}-\phi_{i}\right)>0$. Consider another solution $\boldsymbol{y}$ that is constructed by setting $y_{i}=x_{i}-\epsilon$ and $y_{j}=x_{j}+\epsilon /(n-1)$ for all $j \neq i$. We will show that $\boldsymbol{y}$ belongs to the core and that $\|\boldsymbol{y}-\phi\|<\|\boldsymbol{x}-\phi\|$ which means $\boldsymbol{x}$ is not the fairest core and hence a contradiction.

First, we will show that $\boldsymbol{y}$ is a feasible solution to model (1). We have $\boldsymbol{y}(\mathcal{N})=\boldsymbol{x}(\mathcal{N})=v(\mathcal{N})$. We need to show $\boldsymbol{y}(\mathcal{S}) \geq v(\mathcal{S}), \forall \mathcal{S} \subset \mathcal{N}$. For each coalition $S$, there are two cases:

- $\mathcal{S} \not \ngtr i$, we have $\boldsymbol{y}(\mathcal{S})=\boldsymbol{x}(\mathcal{S})+\epsilon|\mathcal{S}| /(n-1) \geq v(\mathcal{S})$.

- $\mathcal{S} \ni i$, we have,

$$
\begin{aligned}
\boldsymbol{y}(\mathcal{S}) & =\boldsymbol{x}(\mathcal{S})-\epsilon(n-|\mathcal{S}|) /(n-1) \\
& =\boldsymbol{x}(\mathcal{S} \backslash i)+x_{i}-\epsilon(n-|\mathcal{S}|) /(n-1) \\
& =\boldsymbol{x}(\mathcal{S} \backslash i)+\bar{\delta}_{i}-\bar{\delta}_{i}+x_{i}-\epsilon(n-|\mathcal{S}|) /(n-1) \\
& \geq v(\mathcal{S} \backslash i)+\bar{\delta}_{i}+\epsilon-\epsilon(n-|\mathcal{S}|) /(n-1) \\
& \geq v(\mathcal{S})+\epsilon-\epsilon(n-|\mathcal{S}|) /(n-1) \\
& =v(\mathcal{S})+\epsilon(|\mathcal{S}|-1) /(n-1) \geq v(\mathcal{S}) .
\end{aligned}
$$

Here, inequality (6) is due to $x$ belonging to the core and due to our construction of $\epsilon$. Inequality (7) is due to the definition of $\bar{\delta}_{i}$. Thus, $\boldsymbol{y}(\mathcal{S}) \geq v(\mathcal{S}), \forall \mathcal{S} \subset \mathcal{N}$. To complete the proof, we need to show that $\|\boldsymbol{y}-\boldsymbol{\phi}\|<\|\boldsymbol{x}-\boldsymbol{\phi}\|$. We have:

$$
\begin{aligned}
(\boldsymbol{y}-\boldsymbol{\phi})^{t}(\boldsymbol{y}-\boldsymbol{\phi}) & =\left(x_{i}-\epsilon-\phi_{i}\right)^{2}+\sum_{k \neq i}\left(x_{k}+\epsilon /(n-1)-\phi_{k}\right)^{2} \\
& =\sum_{k}\left(x_{k}-\phi_{k}\right)^{2}-\epsilon \frac{n}{n-1}\left(2\left(x_{i}-\phi_{i}\right)-\epsilon\right) \\
& <(\boldsymbol{x}-\boldsymbol{\phi})^{t}(\boldsymbol{x}-\boldsymbol{\phi}) .
\end{aligned}
$$

Here equality (8) results from cancelling most of the terms that appear in the squares and by noticing that $\boldsymbol{x}(\mathcal{N})=\phi(\mathcal{N})=$ $v(\mathcal{N})$. Inequality (9) comes from the fact that $\min \left(x_{i}-\delta_{i}, x_{i}-\phi_{i}\right)=\epsilon>0$. 
Lower bound: The proof for the lower bound is quite similar. Let $\boldsymbol{x}$ be the fairest core and suppose on contradiction that $\epsilon=\min \left(\underline{\delta}_{i}-x_{i}, \phi_{i}-x_{i}\right)>0$. Since $x_{i}<\phi_{i}$, there exists at least another player $j$ such that $x_{j}>\phi_{j}$. Consider another solution $\boldsymbol{y}$ that is constructed by setting $y_{i}=x_{i}+\epsilon$ and $y_{j}=x_{j}-\epsilon$. We will show that $\boldsymbol{y}$ belongs to the core and that $\|\boldsymbol{y}-\boldsymbol{\phi}\|<\|\boldsymbol{x}-\boldsymbol{\phi}\|$ which means $\boldsymbol{x}$ is not the fairest core and hence a contradiction.

We have $\boldsymbol{y}(\mathcal{N})=\boldsymbol{x}(\mathcal{N})=v(\mathcal{N})$. We need to show $\boldsymbol{x}(\mathcal{S}) \geq v(\mathcal{S}), \forall \mathcal{S} \subset \mathcal{N}$. For each coalition $S$, there are two cases:

- $\mathcal{S} \ni i$, we have $\boldsymbol{y}(\mathcal{S})=\boldsymbol{x}(\mathcal{S})+\epsilon\left(1-1_{j \in \mathcal{S}}\right) \geq \boldsymbol{x}(\mathcal{S}) \geq v(\mathcal{S})$, where $1_{j \in \mathcal{S}}$ is equal to 1 if $j \in \mathcal{S}$ and zero otherwise.

- $\mathcal{S} \not \ngtr i$, we have,

$$
\begin{aligned}
\boldsymbol{y}(\mathcal{S}) & =\boldsymbol{x}(\mathcal{S})-\epsilon 1_{j \in \mathcal{S}} \\
& =\boldsymbol{x}(\mathcal{S} \cup\{i\})-x_{i}-\epsilon 1_{j \in \mathcal{S}} \\
& \geq v(\mathcal{S} \cup\{i\})-x_{i}-\epsilon 1_{j \in \mathcal{S}} \\
\geq & v(\mathcal{S})+\underline{\delta}_{i}-x_{i}-\epsilon 1_{j \in \mathcal{S}} \\
& \geq v(\mathcal{S})+\epsilon\left(1-1_{j \in \mathcal{S}}\right) \geq v(\mathcal{S}) .
\end{aligned}
$$

To complete the proof, we need to show that $\|\boldsymbol{y}-\boldsymbol{\phi}\|<\|\boldsymbol{x}-\boldsymbol{\phi}\|$. We have:

$$
\begin{aligned}
(\boldsymbol{y}-\boldsymbol{\phi})^{t}(\boldsymbol{y}-\boldsymbol{\phi}) & =\left(x_{i}+\epsilon-\phi_{i}\right)^{2}+\left(x_{j}-\epsilon-\phi_{j}\right)^{2}+\sum_{k \notin\{i, j\}}\left(x_{k}-\phi_{k}\right)^{2} \\
& =\sum_{k}\left(x_{k}-\phi_{k}\right)^{2}+2 \epsilon\left(\left(\epsilon+x_{i}-\phi_{i}\right)-\left(x_{j}-\phi_{j}\right)\right) \\
& =(\boldsymbol{x}-\boldsymbol{\phi})^{t}(\boldsymbol{x}-\boldsymbol{\phi})-2 \epsilon\left(\left(\phi_{i}-x_{i}\right)-\epsilon\right)-2 \epsilon\left(x_{j}-\phi_{j}\right) \\
& <(\boldsymbol{x}-\boldsymbol{\phi})^{t}(\boldsymbol{x}-\boldsymbol{\phi}) .
\end{aligned}
$$

Here (10) results from the fact that $\left(\phi_{i}-x_{i}\right) \geq \epsilon>0$ and $\left(x_{j}-\phi_{j}\right)>0$.

\section{Numerical Results}

We will demonstrate the properties of the fairest core by comparing its stability, fairness and computation time with that of the core and the Shapley value on randomly generated minimum spanning tree (MST) games. Table 2 shows this comparison for MST games with the number of nodes (players) $n$ varying between 5 and 20 which is shown in the first column. The underlying networks are generated randomly with the densities shown on the second column. Here, a density of $\delta=0.25$ means the chance of having an edge between any two nodes is $25 \%$ while a density of $\delta=1$ means the network is fully connected. The weight of each edge is a uniform number between 0 and 1 . For each choice of $(n, \delta)$ we generate $K$ random instances with $K=50$ for $n \leq 15$ and $K=10$ for $n=20$. We then calculate the Shapley value, the core and the fairest core for each game. The third column measures the percentages of games, among all the random games generated, in which the Shapley value belongs to the core. On average, the Shapley value lies within the core in $23 \%$ of the games. In other words, the Shapley value is unstable in $77 \%$ of the random games generated. We also use the correlation between a solution in the core and the Shapley value as a measure of fairness. Since the core of the MST game is non-empty and is likely non-unique, the correlations might be different among different members of the core. Columns 4-6 show the correlations between three types of core members and the Shapley value to see how 'fair' the solutions are. In column 4, a 'random' core member $\boldsymbol{x}_{r}$ is produced by solving a linear programming feasibility problem that characterises the core by using CPLEX [5], a state-of-the-art optimisation software, with its default settings. Column 5 shows the fairness of the 'unfairest' (or the worst) core member $\boldsymbol{x}_{w}$ - the core member that is farthest from the Shapley value. Column 6 shows the fairness of the fairest core $\boldsymbol{x}_{f c}$ produced by solving model (1). Columns 4-6 show that, on average, a random core member has around 65\% correlation with the Shapley value. However, if we are unlucky and choose the unfairest core, then the correlation is reduced to $29 \%$ (with $19.7 \%$ of the random instances having negative correlations). The fairest core, however, has a very high correlation with the Shapley value (of around 99\%) and this confirms its favourable properties as listed in Theorem 1.

We also measure the computation time for the Shapley value, the core and the fairest core and these are shown in columns 7-9 of Table 2. The Shapley value is calculated by using a brute-force method that averages out all the marginal 


\begin{tabular}{|c|c|c|c|c|c|c|c|c|}
\hline & & \multicolumn{4}{|c|}{ Stability and Fairness } & \multicolumn{3}{|c|}{ Computation time (in seconds) } \\
\hline $\mathrm{n}$ & $\delta$ & $\begin{array}{c}\text { Prob. } \\
(\phi \in \mathcal{C})\end{array}$ & $\begin{array}{c}\text { Corr } \\
\left(\phi, \boldsymbol{x}_{r}\right)\end{array}$ & $\begin{array}{c}\text { Corr } \\
\left(\phi, \boldsymbol{x}_{w}\right)\end{array}$ & $\begin{array}{c}\text { Corr } \\
\left(\phi, \boldsymbol{x}_{f c}\right)\end{array}$ & $\begin{array}{c}\text { Shapley } \\
\text { value }\end{array}$ & Core & $\begin{array}{c}\text { Fairest } \\
\text { core }\end{array}$ \\
\hline \multirow{5}{*}{5} & 0.25 & $62 \%$ & 0.625 & 0.347 & 0.992 & 0.020 & 0.004 & 0.011 \\
& 0.5 & $54 \%$ & 0.709 & 0.216 & 0.988 & 0.016 & 0.004 & 0.006 \\
& 0.75 & $52 \%$ & 0.633 & 0.248 & 0.994 & 0.017 & 0.004 & 0.009 \\
& 1 & $36 \%$ & 0.727 & 0.331 & 0.994 & 0.018 & 0.004 & 0.012 \\
\hline \multirow{1}{1}{10} & 0.25 & $22 \%$ & 0.656 & 0.344 & 0.990 & 0.846 & 0.015 & 0.013 \\
& 0.5 & $20 \%$ & 0.644 & 0.200 & 0.983 & 0.830 & 0.017 & 0.016 \\
& 0.75 & $30 \%$ & 0.660 & 0.299 & 0.988 & 0.826 & 0.017 & 0.011 \\
& 1 & $18 \%$ & 0.678 & 0.291 & 0.989 & 0.834 & 0.018 & 0.014 \\
\hline \multirow{5}{*}{15} & 0.25 & $8 \%$ & 0.661 & 0.293 & 0.985 & 37.79 & 0.822 & 0.080 \\
& 0.5 & $8 \%$ & 0.639 & 0.260 & 0.988 & 37.77 & 0.868 & 0.062 \\
& 0.75 & $8 \%$ & 0.649 & 0.325 & 0.987 & 37.78 & 0.805 & 0.076 \\
& 1 & $12 \%$ & 0.665 & 0.323 & 0.986 & 37.83 & 0.849 & 0.063 \\
\hline \multirow{5}{*}{20} & 0.25 & $0 \%$ & 0.657 & 0.264 & 0.981 & 1592.4 & 37.10 & 1.178 \\
& 0.5 & $20 \%$ & 0.684 & 0.276 & 0.984 & 1587.4 & 37.15 & 0.895 \\
& 0.75 & $10 \%$ & 0.540 & 0.184 & 0.977 & 1589.4 & 38.82 & 1.006 \\
& 1 & $0 \%$ & 0.595 & 0.309 & 0.980 & 1589.9 & 37.13 & 1.070 \\
\hline
\end{tabular}

Table 2: Properties and computation time of the core, the Shapley value and the fairest core.

contributions, i.e. we do not exploit any special property of the games. Thus, the computational complexity for finding the Shapley value grows exponentially with the number of players. Finding a core member involves solving a linear program with an exponentially large number of linear constraints, while finding the fairest core involves solving a quadratic programming with the same underlying set of constraints. At first sight, we might think that finding the fairest core is more computationally involved than finding the core due to the nonlinearity. However, columns 8-9 show that computing the fairest core, assuming the Shapley value is given, actually takes less time than computing the core. Notice that, for MST games, there are very fast methods to construct a core member [3]. However, we take the general approach of using linear programming to demonstrate the result of Theorem 3 for general games. Table 3 shows the computation time of the core and the fairest core in detail. We use a constraint generation framework (see [1], for example, for details about the algorithm) to solve the core and the fairest core. This method is chosen instead of using some off-the-shelf optimisation package because this allows us to explore the relation between the solution times and the calls to the separation routine appeared in Theorem 3. In this framework, we start by solving a relaxed problem with a smaller number of constraints. We then solve the separation problem to check if the optimal solution of the relaxed problem satisfies all the constraints. If that is the case, then the relaxed solution is also the optimal solution of the original problem and we can stop the algorithm. Otherwise, we add the violating constraint to the relaxed problem and continue this process.

We use the same random MST instances generated earlier, and the first two columns of Table 3 include the same set of input on the number of players and the density of the networks. Columns 3-6 show the computational statistics for finding the core, and columns 7-10 show these for the fairest core. The total computation time of the core is shown in column 3, and that of the fairest core is shown in column 7. The total computation time for finding the core is broken down into the time to solve the LP relaxation problems (shown in column 4) and that to solve the constraint generation problems (shown in column 5). The same sets of statistics for finding the fairest core are shown in columns 8 and 9 . The constraint generation problems for finding the core and finding the fairest core are the same, i.e. both of which are equivalent to finding the largest element in a vector of excess values. Notice that, for finding the fairest core, we need to solve quadratic problems, and the total computation time for this is shown in column 8. Although solving a relaxed LP should be easier than solving a relaxed QP with the same size, the computation time for solving the fairest core is shorter than that of the core, because the number of iterations involved (shown in column 10) is much smaller than that for the core (shown in column 6). We expect this reduction in the number of iterations is due to the strict convexity of the objective function in model (1). With that, the total computation time of the fairest core is actually less than that of the core for most of the instances tested. 


\begin{tabular}{|c|c|c|c|c|c|c|c|c|c|}
\hline & & \multicolumn{1}{|c|}{ Computation time of the core } & \multicolumn{4}{c|}{ Computation time of the fairest core } \\
\hline $\mathrm{n}$ & $\delta$ & Total & LP & CG & \# iter. & Total & QP & CG & \# iter. \\
\hline & 0.25 & 0.004 & 0.003 & 0.001 & 4.82 & 0.011 & 0.010 & 0 & 1.82 \\
5 & 0.5 & 0.004 & 0.003 & 0.001 & 4.18 & 0.006 & 0.006 & 0 & 2.02 \\
& 0.75 & 0.004 & 0.004 & 0.000 & 4.66 & 0.009 & 0.009 & 0 & 1.84 \\
& 1 & 0.004 & 0.004 & 0.000 & 4.84 & 0.012 & 0.012 & 0 & 2.26 \\
\hline \multirow{1}{1}{10} & 0.25 & 0.015 & 0.013 & 0.002 & 22.82 & 0.013 & 0.013 & 0 & 3.24 \\
& 0.5 & 0.017 & 0.015 & 0.001 & 22.9 & 0.016 & 0.016 & 0 & 3.74 \\
& 0.75 & 0.017 & 0.014 & 0.002 & 24.12 & 0.011 & 0.011 & 0 & 3.24 \\
& 1 & 0.018 & 0.018 & 0.000 & 22.94 & 0.014 & 0.013 & 0 & 3.58 \\
\hline & 0.25 & 0.822 & 0.647 & 0.159 & 126.3 & 0.080 & 0.075 & 0.005 & 5.76 \\
15 & 0.5 & 0.868 & 0.708 & 0.149 & 133.82 & 0.062 & 0.055 & 0.006 & 4.6 \\
& 0.75 & 0.805 & 0.652 & 0.142 & 125.9 & 0.076 & 0.066 & 0.010 & 5.36 \\
& 1 & 0.849 & 0.696 & 0.144 & 129.1 & 0.063 & 0.059 & 0.004 & 5 \\
\hline \multirow{3}{*}{20} & 0.25 & 37.10 & 3.579 & 33.49 & 307.2 & 1.178 & 0.119 & 1.059 & 9 \\
& 0.5 & 37.15 & 3.702 & 33.38 & 305 & 0.895 & 0.086 & 0.810 & 6.8 \\
& 0.75 & 38.82 & 3.764 & 34.99 & 320.6 & 1.006 & 0.064 & 0.942 & 7.7 \\
& 1 & 37.13 & 3.619 & 33.44 & 306.1 & 1.070 & 0.114 & 0.956 & 8.4 \\
\hline
\end{tabular}

Table 3: Computation time of the Shapley value and the fairest core.

\section{Acknowledgement}

Research support from the LANCS Initiative is gratefully acknowledged. The author also thanks Talal Rahwan, Long Tran-Thanh, Philip Le, and participants of the coalitional structure generation group at the University of Southampton for helpful comments and discussions.

\section{References}

[1] G. Desaulniers, J. Desrosiers, and M.M. Solomon. Column generation, volume 5. Springer, 2005.

[2] S.H. Gow and L.C. Thomas. Interchange fees for bank atm networks. Naval Research Logistics (NRL), 45(4):407-417, 1998.

[3] Daniel Granot and Gur Huberman. On the core and nucleolus of minimum cost spanning tree games. Mathematical Programming, 29(3):323-347, 1984.

[4] M. Grotschel, L. Lovász, and A. Schrijver. Geometric Algorithms and Combinatorial Optimizations. Springer-Verlag, 1993.

[5] ILOG Inc. Cplex 7.1 user's manual.

[6] M.K. Kozlov, S.P. Tarasov, and L.G. Khachiyan. The polynomial solvability of convex quadratic programming. USSR Computational Mathematics and Mathematical Physics, 20(5):223-228, 1980.

[7] R. Lindelauf, H.J.M. Hamers, and B.G.M. Husslage. Game theoretic centrality analysis of terrorist networks: the cases of jemaah islamiyah and al qaeda. Tilburg University, 2011.

[8] D.C.R. Monteiro and I. Adler. Interior path following primal-dual algorithms. part ii: Convex quadratic programming. Mathematical Programming, 44(1-3):43-66, 1989.

[9] L.S. Shapley. A value for n-person games. in Kuhn, H. and Tucker, A.W., Contributions to the Theory of Games II, pages 307-317, 1953.

[10] L.S. Shapley. Cores of convex games. International journal of game theory, 1(1):11-26, 1971. 